\title{
Sustainable Manufacturing: Metrics, Standards, and Infrastructure - Workshop Summary
}

\author{
Sudarsan Rachuri, Ram D. Sriram, Anantha Narayanan, Prabir Sarkar, \\ Jae Hyun Lee, Kevin W. Lyons, and Sharon J. Kemmerer, NIST, Member, IEEE
}

\begin{abstract}
This report summarizes the presentations, discussions, and recommendations of the National Institute of Standards and Technology (NIST) Workshop "Sustainable Manufacturing: Metrics, Standards, and Infrastructure” held at NIST, Gaithersburg, Maryland, USA, October 13 through October 15, 2009. The primary objective of this Workshop was to bring together experts and various stakeholders to identify and discuss measurement and standards enablers that positively affect the social, economic, environmental, and technological aspects of designing sustainable production processes and products. The Workshop was well attended and consisted of thirty presentations organized under five sessions: 1) Government Initiatives; 2) Industry Perspectives; 3) University Research; 4) Non-Government Organizations (NGOs) research; and 5) Solution Provider's Views. Two breakout sessions and an industry panel provided a set of recommendations for addressing critical issues in sustainable manufacturing.
\end{abstract}

\section{INTRODUCTION}

$\mathrm{N}_{\mathrm{s}}^{\mathrm{e}}$ ext generation product design and manufacturing will be strongly influenced by life cycle environmental impacts and resource depletion. Sustainable manufacturing is a systems approach for the creation and distribution (supply chain) of innovative products and services, that: minimizes resources (inputs such as materials, energy, water, and land); eliminates toxic substances; and produces zero waste that in effect reduces green house gases, e.g., carbon intensity, across the entire life cycle of products and services.

Hence, sustainable manufacturing practices will play an important role in "meeting the needs of the present without compromising the ability of future generations to meet their own needs.”[1] Sustainable manufacturing is causing companies to implement new design and analysis procedures, energy reduction methods, material reduction efforts, and improved materials handling practices. Thus, minimizing environmental impact has become a critical manufacturing industry requirement throughout the product life cycle. To foster sustainable practices, there needs to be a measurement methodology to assign the energy and environmental cost at each stage in that life cycle. Information must be available at

Manuscript received April 9, 2010.

Sudarsan Rachuri*, Ram D. Sriram, Anantha Narayanan, Prabir Sarkar, Jae Hyun Lee, Kevin W. Lyons, and Sharon J. Kemmerer are with the National Institute of Standards and Technology, Gaithersburg, MD 20899 USA (*corresponding author to provide phone: 301-975-4264; fax: 301-975-4635; e-mail: \{rachuri.sudarsan, ram.sriram, anantha.narayanan, sarkar.prabir, lee.jaehyun, kevin.lyons, sharon.kemmerer\} @ nist.gov). the early design stage about the ultimate costs of each design decision for a new product, and the decisions themselves must be available at the end of product life to ascertain how to properly dispose of or reclaim the components.

Ensuring a sustainable future requires an integrated system of systems approach. Interlinked pathways of interaction at various levels characterize such systems. These levels span technical, economic, ecological, and societal issues. The interactions within and across these levels are critical to the fundamental understanding of sustainable design and manufacturing, because tackling any one of the issues in isolation could result in unintended consequences.

The systems approach of sustainability requires life cycle thinking. The life cycle of a product starts with raw material extraction and processing, continues with the pre-design and fabrication of the relevant semi-finished products, includes manufacturing and assembly of the final product as well as its transportation, use and maintenance, and concludes with the end-of-life operations. This last stage includes recycling of materials and, after adequate treatment, final disposal of waste.

\section{OVERVIEW OF THE WORKSHOP}

The Workshop "Sustainable Manufacturing: Metrics, Standards, and Infrastructure" was held at NIST, Gaithersburg, Maryland, USA, October 13 through October 15, 2009 [2].

\section{A. Objective}

The primary objective of this Workshop was to bring together experts and various stakeholders to identify and discuss measurement and standards' enablers that positively affect the social, economic, environmental, and technological aspects of designing sustainable production processes, products, and services.

The Workshop consisted of technical sessions (which included three keynotes), breakout discussions, and industrial showcases that addressed important issues necessary for the production of sustainable systems.

The topics for the technical sessions included (subtopics are given as examples):

1. Develop General Notion of sustainable manufacturing

- Including indicators, indices, metrics for sustainability, Sustainable Manufacturing Maturity Model, macro 
level and micro level sustainability, the notion of triple bottom line, and corporate social responsibility.

2. Design of sustainable products, services, and manufacturing systems

- Integrating environmental aspects into product design and development, design for process and product sustainability, product lifecycle management and life cycle analysis, material science, advanced manufacturing technologies, nano-manufacturing, energy efficiency, conservation for production and use of products, reduce, reuse, and recycling, information infrastructure including advanced models and semantics for product and process, and manufacturing simulation.

3. Establish standards and industry best practices for sustainable systems

- To include standards landscape for product, process representation, national and international standards and regulations for sustainability (e.g., ISO 14000, RoHS (Restriction of Hazardous Substances) [3], REACH (Registration, Evaluation, Authorization and Restriction of Chemical substances) [4], and WEEE (Waste Electrical and Electronic Equipment)) [5], risk analysis of policy instruments (cap and trade), regulations, and cost of compliance.

4. Develop next generation information and communication technologies (ICTs) for sustainable manufacturing

- ICT for design, manufacturing, and supply chain optimization for sustainable manufacturing.

- Large scale data modeling and semantic technologies for sustainable manufacturing.

- Tools, standards, and industry best practices for sustainable systems.

- Interoperability among PLM (Product Lifecycle Management) and LCA (Life Cycle Assessment) tools to support energy and material monitoring and saving.

\section{B. Presentation Summaries}

The Workshop consisted of three keynote presentations, five technical sessions, two breakout discussions, and an industrial panel discussion that addressed important issues necessary for the production of sustainable systems. The keynote speakers included Mary Saunders, Assistant Secretary Manufacturing and Services, International Trade Administration (ITA), Department of Commerce; Mark Cohen, Vice President for Research at Resources for the Future; and Bob Bechtold, CEO of HARBEC Plastics Inc.. Mary Saunders described recent developments in the Sustainable Manufacturing Initiative of the Manufacturing and Services (MAS), while Mark Cohen gave an overview on sustainability reporting and the Global Reporting Initiative (GRI). Bob Bechtold presented a case study of small and medium-sized enterprises (SMEs) and lessons learned from implementing sustainability practices.

The five technical sessions consisted of thirty presentations, organized into five sessions: 1) Government Initiatives; 2) Industry Perspectives; 3) University Research; 4) Non-Government Organizations (NGOs) Research; and 5) Solution Providers' Views. Sustainable manufacturing initiatives at the National Science Foundation (NSF), National Institute of Standards and Technology (NIST), and National Aeronautics and Space Administration (NASA) were presented in the Government Initiatives session. The Industry Perspectives session involved participation from industry leaders, focusing on the costs, benefits, and challenges in incorporating sustainability in the industry. The session had presentations from various industries, including Ford, GM, GE Aviation, Lockheed Martin, Rockwell Automation, P\&G, Xerox, URS, and Masco Retail Cabinet Group. The University Research session provided Workshop participants an opportunity to discuss academic research issues in sustainable manufacturing. This session included presentations from Rochester Institute of Technology, Purdue University, University of Kentucky, Stanford University, Georgia Institute of Technology, Portland State University, and Texas Tech University. The Non-Government Organizations Research session involved participation from various NGOs, Standards Developing Organizations (SDOs), and industry consortia, focusing on various standards development efforts, harmonization of standards, and sustainability reporting mechanisms and standards. The American National Standards Institute (ANSI), American Society for Testing and Materials (ASTM), World Resource Institute (WRI), National Center for Manufacturing Sciences (NCMS), National Council For Advanced Manufacturing (NACFAM), and Cadmus Group gave presentations in this session. The Solution Providers session predominantly focused on the need for tool support for sustainable manufacturing, currently available tool support, and the standards compliance of the applications presented. Companies are becoming increasingly interested in adhering to standards such as RoHS, REACH, and WEEE to compete globally. Major software solution providers, such as Parametric Technology Corporation (PTC), Digital Enterprise Lean Manufacturing Interactive Application (DELMIA), Siemens PLM Solutions, and Siemens R\&D presented their suite of software tools and their current capabilities and future extensions.

\section{Breakout Sessions}

The breakout sessions consisted of two groups:

1. Critical factors driving sustainable manufacturing: The themes discussed in this group were business case for sustainability, promoting eco-innovation, standards and metrics, tool support, promoting reduced energy consumption, and positioning of standards.

2. Decision support system for sustainable manufacturing: The themes discussed in this group were standards harmonization, science of sustainability, greening the supply chain, data availability, and needs. 
The participants generated recommendations or conclusions by selecting the best ideas or combining ideas for the "top attributes" identified. Each group covered as many of the "top attributes" as possible.

\section{Group 1: Critical factors driving sustainable manufacturing}

Group 1 took up the following set of key issues and subtopics for further elaboration, starting with this set and identifying the most common themes.

1. Business: How do the following diverse factors affect the business case: compliance, economic models, profit, case studies, and new sustainable products?

2. Eco-innovation: How to promote eco-innovation?

3. Metrics: How to develop metrics based on solid models, cases, and good data for model validation?

4. Tools: What are the gaps and problems with a methodology such as LCA? What is the business case for tools?

5. Energy: How to reduce energy consumption for sustainable growth?

6. Standards: How should standards be posed so that the positive incentive side drives them?

Multiple factors affecting sustainable products: Businesses need to make decisions based on a diverse range of factors when it comes to sustainable manufacturing. To make sound decisions, they must consider compliance, economic models, profit, and case studies.

Problem or issue:

- How to make sound business decisions when confronted with a variety of sustainability factors? Root cause:

- Businesses are mainly concerned with profit, sales, and market share. It is difficult to compare these factors among different diverse companies and industries.

Recommendation:

- Develop a better understanding of the drivers and relationships among these factors.

Action plans:

- Reduce costs by analyzing the life cycle of products, establishing a link between sustainability and financial performance.

- Develop measurable indices, which can be represented in monetary terms. This must assist decision making by comparing profits with cost of being sustainable. Much of the responsibility to investigate the science behind these indices and establish a methodology for their measurement lies with academia.

- Anticipate regulations and clearly identify the minimum requirements that satisfy compliance. NGOs have the responsibility to educate the industry and society at large about standards and publish business cases illustrating them.

- Towards this end, we expect the industry to be transparent and encourage sharing and co-operation. Governments must provide a level playing field for all businesses.

Promoting eco-innovation: Eco-innovation is the innovative design of products and processes that are sustainable or contribute to sustainable development. The quest for sustainable products has provided an opportunity to come up with innovative designs for the future. However, such designs have so far been risky and under-funded.

Problem or issue:

- How to promote eco-innovation?

Root cause:

- There is a lack of funding for fundamental research in the design of sustainable products. Research in this area requires a high amount of resources and has a high uncertainty.

Recommendation:

- Be open minded, and learn from previous experiences in Europe.

Action plans:

- Learn a lot about eco-innovation from Europe, where a number of innovative designs have been developed in recent years for green and sustainable products.

- Encourage open-mindedness. Develop new business cases for innovative designs, and facilitate their acceptance.

- Increase industry-academia interaction and find avenues for funding for eco-innovation.

Metrics, data models, and validation: There is a need for simple and high level metrics, and these need to be supported by good data models. Previous attempts have led to metrics that are difficult to estimate, and for which data is not readily available.

Problem or issue:

- How to develop metrics based on well-defined data models, and where can we get good data to validate them?

Root cause:

- There is no standard single unit for comparing metrics, making them difficult to compare and analyze. Recommendation:

- Develop simple high-level metrics.

Action plans:

- Identify a multi-level approach, leading to simple metrics at the highest level.

- Ensure that it is easy to compare the metrics to standards.

- Metrics must be normalized with respect to production. 
Methodologies in practical use: The focus here is on the use of methodologies such as LCA when applied to the supply chain.

Problem or issue:

- What are gaps and problems with a methodology such as LCA?

Root cause:

- Not all the companies, especially small companies, in the supply chain can apply the methodologies to their products.

o They may not have enough information.

o They may not have an efficient way to access information.

o They may not have enough power to control their sub-tier suppliers to get information.

Recommendation:

- Develop a simple and transparent methodology for small companies to go through it in a short time and less cost.

- Create a new business model for companies to conduct the methodology voluntarily in pursuit of their profits.

Action plans:

For Academia

- Develop transparent methodologies, which:

o Can compute sustainability without exposing critical technology of the companies.

o Can build different levels of information and process models in supply chain.

o Include a role of auditing and validating.

- Develop clear metrics for the methodology.

For industry

- Create a new business model.

Necessities for reducing energy consumption: Companies hesitate to invest money for improved energy efficiency of their manufacturing process (e.g., investment on energy-efficient facilities for replacing legacy facilities). The government needs to have a proper carrot and stick approach that encourages companies' investment. Current methods to evaluate energy consumption and the lack of decision-support tools for this were addressed. The evaluation methods and decision-support tools are critical for companies to decide their investment. The evaluation methods should be able to calculate the environmental impacts, and these evaluation methods should consider the energy life cycle and source types. Additionally, there is a need for developing energy simulation models and analysis tools for a trade-off analysis between investment and environmental impacts.

Problem or issue:

- How to recognize and promote reducing energy consumption for sustainable growth?

- Why do companies struggle to make a decision to Root cause: invest money to reduce energy consumption?

- Current evaluation methods for energy consumption are not sufficient to measure environmental impacts.
- Decision-support tools are necessary for companies to make their decision to invest money to reduce energy consumption.

Recommendation:

- Develop a carrot and stick approach, which encourages a company to invest money to reduce the amount of energy consumed.

- Create eco-labeling for manufacturing machines.

Action plans:

- Develop evaluation methods for energy consumption which consider:

o Source types of energy.

o Life cycle of energy.

o Depreciation of assets over time with respect to energy spent.

o Develop decision-support tools that can simulate systems that use energy.

o Evaluate the trade-off between investment and environmental impacts.

- Develop best-practices in industry to

o Reduce resource consumption.

o Recycle energy.

Standards: Simple and credible metrics are essential for sustainability standards to hold a strong market position. If a standard includes simple and representative metrics, it will be used by more companies (refer to the metrics category in this breakout session summary). In addition to the simple metrics issue, sustainability standards need to have brands of conformity associated with them. The brand quality of the standards should be maintained. A branded sustainability standard can be a positive driver of the market. If the brand is well known in the market, companies will invest money to get a certification or award of the standard.

Problem or issue:

- How should standards be posed in the market so that the positive incentive side drives them?

Root cause:

- Metrics in the standards are too complex for companies to use.

- Current sustainability standards may not have strong effects on market share.

Recommendation:

- (See the recommendation about metrics.)

- Create brand values of sustainability standards. Action Plans:

- (See the action plans in the metrics category.)

- Create brand values of sustainability standards.

o Associate awards or certifications of the standards to sustainable products.

- Maintain the brand values of sustainability standards.

Group 2: Decision support system for sustainable manufacturing

Group 2 considered the following areas: Alignment of sustainability initiatives between US/EU/World, at local, 
state, and federal levels; sustainability product labeling and grading; standards, metrics, indicators, and standards' landscape for the enterprise; greening the supply chain; information modeling, semantic technologies, tools to support a systems approach; data availability and needs; education and outreach; cost of compliance and reporting; mathematical models and science of sustainability; and making a business case for sustainability. In the final deliberations, Group 2 focused on the following themes: 1. Harmonization of standards, 2. Mathematical models and science of sustainability, 3. Greening the supply chain, and 4. Data availability and needs.

Harmonization of standards: The current state of the art in sustainability standards is that there are too many standards out there, and there is no proper organization or association between them. It is difficult for businesses to make sense of the large number of standards, identifying which ones are relevant to them, or handle overlapping concerns between different standards. Some guidance is needed in choosing the right standards that are of common concern to the industry.

Problem or issue:

- How can we harmonize different standards? How can we identify the most important standards?

Root cause:

- There are too many standards, metrics, and definitions for sustainability.

Recommendation:

- Several recommendations were made, including the following:

o Allow the market to decide - let businesses choose what standards they follow.

o The government could choose a set of standards that all businesses must follow.

o The original equipment manufacturers (OEMs) might follow the strictest standards. In most cases, this would entail conformance to less demanding standards.

Action plans:

- The participants of Group 2 agreed that this is a difficult issue to address, and there can be no definitive action plan at this stage. Some recommendations were made, and various market factors will affect the outcome.

Mathematical models and science of sustainability: Strong mathematical models must support decision support systems for sustainable development. The science behind sustainability metrics and recommendations must be strongly grounded.

Problem or issue:

- How can we develop strong mathematical models and scientific studies for sustainability?

Root cause:

- Need for strong mathematical models for sustainability.

Recommendation:
- Develop open source models that are generic, extensible, and easy to build and share.

Action plans:

- Stimulate open source models from research institutions. Industries can play a collaborative role by providing test cases.

- Recognize data exchange problems and create exchange protocols. NIST could play the role of a central repository for data exchange protocols, and the definition of a data exchange specification.

- Separate modeling into various levels of detail.

Data availability and needs: One of the main hindrances to activities such as LCA is the lack of readily available data. Several methodologies related to sustainability suffer from a shortage of data. It is difficult to conduct research and develop new methodologies without access to data.

Problem or issue:

- What are the data needs and how can they be satisfied? Root cause:

- It is hard to get data due to companies' privacy policies, and the available data is usually inaccurate and error prone.

Recommendation:

- Create global data repositories.

- Address aggregation and disaggregation of data. Action plans:

- Identify the stakeholders to address data aggregation and disaggregation problems.

- Address data needs for conformance assessment.

- Collect activity based data (such as water, energy).

- Collect data globally and use global repositories.

Greening the supply chain: A commonly faced problem throughout the industry is sustainability analysis and compliance management in the supply chain. Most OEMs find that their supply chains are unaware of sustainability standards or do not have any data for compliance management. Often, their contracts mention nothing related to sustainability and require no action by the supplier to be compliant. This is changing slowly with initiatives by businesses such as Wal-Mart.

Problem or issue:

- How can we ensure that the supply chain is compliant? Root cause:

- Supply chains have no sustainability concerns.

- Small and medium companies lack supplier information.

Recommendation:

- Develop reporting standards for suppliers.

- Industry must drive suppliers to be compliant.

Action plans:

- Provide education and training to suppliers in simple terms, stressing on the importance of compliance.

- Develop standards for suppliers to report data to OEMs. OEMs can prioritize the scope of the data in conformance with standards. 
- Industry must drive the suppliers to be conformant with sustainability standards.

- Develop a database of commonly available information on material content, processes etc.

\section{CONCLUSION REMARKS}

The primary objective of the Workshop was to bring together experts and various stakeholders to identify and discuss measurement and standards enablers that positively affect the social, economic, environmental, and technological aspects of designing sustainable production processes and products. The Workshop was well attended with thirty presentations organized into five sessions: 1) Government Initiatives; 2) Industry Perspectives; 3) University Research; 4) Non-Government Organizations (NGOs) research; and 5) Solution Providers' Views.

The Workshop participants identified several challenges faced by the manufacturing industry in its pursuit of sustainability goals and provided a set of key recommendations. The major challenges identified were as follows:

1. Industry is unable to measure economic, social, and environmental consequences of their activities and products accurately during the entire life cycle and across their supply chain. One of the main reasons for this is the lack of data traceable to a neutral organization. Even if the data were available, industry is finding it difficult to aggregate and disaggregate data to compute sustainability metrics.

2. Full life cycle analysis or assessment of products requires new methods to analyze, integrate, and aggregate information across hierarchical levels, organizational entities, and supply chain participants.

3. Industry lacks neutral and trusted standards and programs to demonstrate, deploy, and accredit new sustainable manufacturing practices, guidelines, and methods.

4. There are too many metrics; they need consolidation and harmonization. Also, they need to be 'monetized' as appropriate.

5. Regulations need to be supported by industry standards (e.g., RoHS and IPC-1752). These regulations and standards should be harmonized.

6. Information standards are necessary to enable interoperability among engineering tools, business enterprise tools and Life Cycle Assessment tools for an integrated systems approach.

Key recommendations from the Workshop participants follow, in no particular order.

Metrics (or indicators):

- Pursue a multi-level approach for metrics, with simple metrics at the highest level.

- Consolidate and harmonize the diverse set of existing metrics.

- Monetize metrics as appropriate.
- Support regulations (e.g., RoHS) with industry standards (such as IPC-1752).

- Develop a strategy for the harmonization of many standards and directives that currently exist for sustainability.

- Create brand values for sustainability standards and maintain the brand values.

\section{Infrastructure:}

- Create a software infrastructure for gathering, analyzing, exchanging, and aggregating information for sustainability, including support for global data repositories.

- Develop a simple and transparent methodology for life cycle assessment calculation.

- Develop a science of sustainability, including open source models that are generic, extensible, verifiable, and easy to build and share.

\section{Best Practices:}

- Create a new business model for companies to apply the methodology developed for LCA voluntarily, which maximizes profits while minimizing costs.

- Develop best practices for eco-innovation, i.e., design of products and processes that are sustainable or contribute to sustainable development.

- Create eco-labeling for manufacturing processes and machines.

- Develop sustainability reporting standards for suppliers, and provide education and training to suppliers in simple terms, stressing the importance of compliance.

- Develop traceable life cycle inventory data to enable life cycle analysis of products, processes, and services and to enable verification and validation of life cycle impact measurements and benchmarking.

\section{DISCLAIMER}

No approval or endorsement of any commercial product by the National Institute of Standards and Technology is intended or implied. Certain commercial equipments, software, instruments, or materials are identified in this report to facilitate better understanding. Such identification does not imply recommendations or endorsement by the National Institute of Standards and Technology, nor does it imply the materials, software, or equipment identified are necessarily the best available for the purpose.

\section{REFERENCES}

[1] Our Common Future: Report of the World Commission on Environment and Development, Oxford University Press, 1987.

[2] S. Rachuri, R. D. Sriram, A. Narayanan, P. Sarkar, J.H. Lee, K. W. Lyons, and S. J. Kemmerer, "Sustainable manufacturing: metrics, standards, and infrastructure - NIST Workshop report," National Institute of Standards and Technology (NIST), Gaithersburg, MD, USA, NIST interagency/internal report (NISTIR) 7683, 2010

[3] RoHS homepage. [Online] Available: http://www.rohs.gov.uk/.

[4] REACH homepage. [Online] Available: http://ec.europa.eu/enterprise/sectors/chemicals/reach/index_en.htm.

[5] European Commission WEEE page. [Online] Available: http://ec.europa.eu/environment/waste/weee/index_en.htm. 